\title{
Cycle to Cycle Variation Study in a Dual Fuel Operated Engine
}

\author{
ShyamSundar Pasunurthi and Ravichandra Jupudi
}

GE, Global Research Center

\section{Sameera Wijeyakulasuriya}

Convergent Science Inc.

\section{Sreenivasa Rao Gubba}

GE, Global Research Center

\section{Hong Im and Mohammed Jaasim Mubarak Ali}

King Abdullah University of Science and Technology

\section{Roy Primus and Adam Klingbeil}

GE, Global Research Center

\section{Charles Finney}

Oak Ridge National Laboratory

CITATION: Pasunurthi, S., Jupudi, R., Wijeyakulasuriya, S., Gubba, S. et al., "Cycle to Cycle Variation Study in a Dual Fuel Operated Engine," SAE Technical Paper 2017-01-0772, 2017, doi:10.4271/2017-01-0772.

Copyright (C) 2017 SAE International

\begin{abstract}
The standard capability of engine experimental studies is that ensemble averaged quantities like in-cylinder pressure from multiple cycles and emissions are reported and the cycle to cycle variation $(\mathrm{CCV})$ of indicated mean effective pressure (IMEP) is captured from many consecutive combustion cycles for each test condition.

However, obtaining 3D spatial distribution of all the relevant quantities such as fuel-air mixing, temperature, turbulence levels and emissions from such experiments is a challenging task.

Computational Fluid Dynamics (CFD) simulations of engine flow and combustion can be used effectively to visualize such 3D spatial distributions. A dual fuel engine is considered in the current study, with manifold injected natural gas (NG) and direct injected diesel pilot for ignition. Multiple engine cycles in $3 \mathrm{D}$ are simulated in series like in the experiments to investigate the potential of high fidelity RANS simulations coupled with detailed chemistry, to accurately predict the CCV.
\end{abstract}

Cycle to cycle variation (CCV) is expected to be due to variabilities in operating and boundary conditions, in-cylinder stratification of diesel and natural gas fuels, variation in in-cylinder turbulence levels and velocity flow-fields. In a previous publication by the authors [1], variabilities in operating and boundary conditions are incorporated into several closed cycle simulations performed in parallel. Stochastic variations/stratifications of fuel-air mixture, turbulence levels, temperature and internal combustion residuals cannot be considered in such closed cycle simulations. In this study, open cycle simulations with port injection of natural gas predicted the combined effect of the stratifications on the $\mathrm{CCV}$ of in-cylinder pressure. The predicted Coefficient of Variation (COV) of cylinder pressure is improved compared to the one captured by closed cycle simulations in parallel.

\section{Introduction}

The availability of natural gas reserves has led to a growing interest in the development of fuel-flexible 'dual-fuel' (DF) engines that can operate on diesel as well as natural gas. In such engines, diesel fuel can be as low as one percent of the total fuel energy at full-power operation [2] . In such a scenario, diesel fuel acts as a pilot ignition source to the combustion of pre-mixed NG. However, this generally requires lowering the compression ratio of the diesel engine and changing the existing diesel injector to a micro-pilot diesel injector, sacrificing the ability to run full power on only diesel. Another alternative is to continue operating with a standard diesel injector but

This manuscript has been authored by UT-Battelle, LLC under Contract No. DE-AC05-00OR22725 with the U.S. Department of Energy. The United States Government retains and the

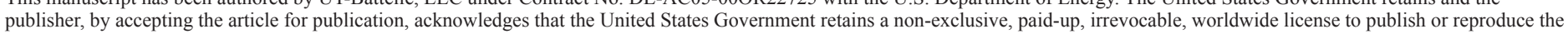

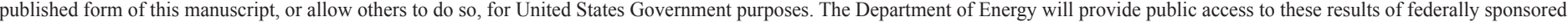
research in accordance with the DOE Public Access Plan (http://energy.gov/downloads/doe-public-access-plan). 
inject lower quantities of diesel. This preserves the ability of the engine to produce full power on $100 \%$ diesel and only requires retrofitting with the NG fuel system and controls for DF operation.

In DF engines, when operated in lean burn mode but rich enough to support flame front propagation, combustion begins with autoignition of diesel fuel that is injected into the engine cylinder. Combustion of NG is initiated by the radicals from the dissociation and combustion of diesel vapor, resulting in a flame front propagating through the remaining unreacted gas mixture. The 'quality' of the combustion (i.e., how much of the released energy is available to generate piston work and mitigation of unwanted emissions) depends on the speed, phasing and completeness of the fuel energy release in the high pressure and temperature environment.

The COVof IMEP (Indicated Mean Effective Pressure) is generally used to indicate the magnitude of CCV. CCV is also referred to as 'cyclic variability' or 'cyclic dispersion', as the inherent differences within the cylinder may produce as well as reflect large pressure variation from one engine cycle to the next. Alternatively, understanding the cause and effect of $\mathrm{COV}$ of in-cylinder pressure would also help to find the ways to mitigate the CCV.

Typically, CCV is higher in dual fuel engines than in diesel engines. Various reasons for this include non-optimal performance of the diesel injector (variability in relative magnitude of mass injected, variability in start of diesel injection, variability in injection duration, fuel nozzle hole-to-hole variability in diesel injected) [ $\underline{3}-\underline{4}]$, variability in the amount of trapped NG, stratification of NG in the cylinder [ $\underline{5}-\underline{6}]$ and the variability in the operating and boundary conditions (pressure, temperature and internal residuals). All these potentially affect the start, extent (depending on trapped NG) and rate of the combustion of pre-mixed NG and thus lead to typically higher $\mathrm{CCV}$ than in diesel engines. Higher CCV generally results in reduced fuel economy and engine performance, limiting the practical operating range of the engine in real-world applications.

Experimental uncertainties and variations in the operating parameters, operating conditions and operation of components like the diesel injector at off-design or off-peak load conditions can be expected to lead to higher COV of IMEP. The stochastic nature of pre-mixing of air and NG as well as the diesel spray at low fueling levels can also lead to higher COV of IMEP.

Computational Fluid Dynamics (CFD) simulations assisted by supercomputing facilities can be used effectively to establish design methodologies and short-list design options. High-fidelity CFD can also provide guidance and focus to single- and multi-cylinder engines testing and reduce hardware iterations. Engine manufacturers are now investing in CFD to understand combustion instabilities using single-and/or multiple-cycle simulations. A major challenge is to capture the subtle and infrequent system dynamical features which span the spatio-temporal domain. Capturing the nonlinear feedback from prior engine cycles that affect combustion stability requires simulating many sequential engine cycles. For experimental engine studies, recording thousands of consecutive cycles is a standard capability for many spatially averaged quantities, but it is still not practical to do the same in CFD. However, in the current study, a series of several full-cycle Reynolds Averaged Navier Stokes (RANS) simulations were performed. The simulations enable the study of the stochastic aspect of naturally induced stratification in (a) temperature, (b) turbulence and (c) recirculated combustion products from the previous cycle as internal residuals and (d) the impact on the NG distribution within the cylinder.

\section{Computational Setup}

\section{Numerical Model}

The computational fluid dynamics solver CONVERGE version 2.3.6 [7] was used in this study. Adaptive mesh refinement (AMR) based on the curvature (second derivative) of shear and normal components of velocity and temperature is used to resolve flow spray and propagating flames in the engine.

In this study, a second-order-accurate spatial discretization scheme and fully-implicit first-order-accurate time integration scheme were used for the governing conservation equations. The solver uses a modified cut-cell Cartesian method that allows the use of simple orthogonal grids. The orthogonality of the grid simplifies the numerics and allows second-order accuracy to be maintained without enforcing expensive corrections for cell-face realignment. Some faces of boundary cells are cut by the boundary and may not align with the coordinate directions. When the boundaries move (e.g., piston, valves) during a given time step, the boundary cells are recut, thus creating new boundary faces. The mesh away from moving boundaries do not get altered/morphed. This procedure eliminates mesh deformation due to boundary motion and minimizes numerical diffusion. It should also be noted that the code switches to a first-order up-winded scheme if dispersion in velocity, density or temperature beyond a user specified value is detected. This is conducted on a cell face-by-cell face basis and is needed to maintain solution stability, while minimizing the reduction of solution accuracy. The transport equations are solved using the pressure implicit with splitting of operators method (PISO) [] $]$. The renormalization group (RNG) k- $\varepsilon$ turbulence model [9] was used to model turbulence, with a standard wall function at the walls. High-fidelity RANS simulations can also capture cyclic variation if all RANS length scales are resolved. The smallest length scales under typical engine conditions range from 1-10 $\mu \mathrm{m}$. However, with the added turbulent viscosity from a RANS turbulence model, these smallest length scales are in the order of 0.1-1.0 mm [10]. This means that to resolve all length scales in a RANS simulation, a grid resolution in the order of $0.1 \mathrm{~mm}$ is sufficient. Due to the added numerical viscosity from the RANS model, all length scales smaller than in the order of $0.1 \mathrm{~mm}$ has disappeared and cannot be resolved even if the mesh is refined below $0.1 \mathrm{~mm}$. Therefore, a RANS simulation would not realize to a DNS simulation with sufficient grid resolution. It has been shown previously that grid resolutions in the order of $0.25 \mathrm{~mm}$ is sufficient to model turbulent combustion in diesel engines [11], gasoline engines [12], gasoline and diesel dual-fuel engines [13], and in natural gas and diesel dual-fuel engines [14]. Using 20 consecutive cycles, Scarcelli and Sevik [12] showed how RANS simulations can be effectively used to represent cyclic variability for EGR dilute and non-dilute gasoline engines. This work by Scarcelli and Sevik [12] used an earlier version of the same numerical code and similar spray, turbulence, combustion and mesh resolution settings as used in the current work. 
The solver uses various time-step controls to ensure that important flow parameters (e.g. CFL number, combustion rate, spray penetration, liquid evaporation) are kept below user-specified maximum values. The code automatically adjusts the time step when deviation from the set limits is sensed. This was an important feature to have for the problem analyzed, to accurately resolve the different (and sometimes competing) physical phenomena involved. The computations were run in parallel on distributed memory machines using the message passing interface (MPI).

The simulations were run on a full cylinder geometry (not a sector) with intake and exhaust manifolds. Natural gas (NG) inlet in the intake manifold corresponds to the same location as in the experiment (Figure 1a). The maximum cell count reached in the simulation was 6.5 million, which occurred when spray embedding (fixed grid refinement around spray cone) and AMR are operational during the diesel spray (Figure 1b). If the entire computational domain was to be refined to the smallest cell size used to resolve turbulent flame propagation the total cell count required would have been $\sim 122$ million, illustrating the benefit of using AMR. The full cycle simulations were simulated on a cluster at KAUST [24] using 240 cores.

Advanced load balancing algorithm 'METIS' is employed in the current work. A recent study [14] showed 45-65\% speedup using METIS for a GDI simulation using the same numerical tool.

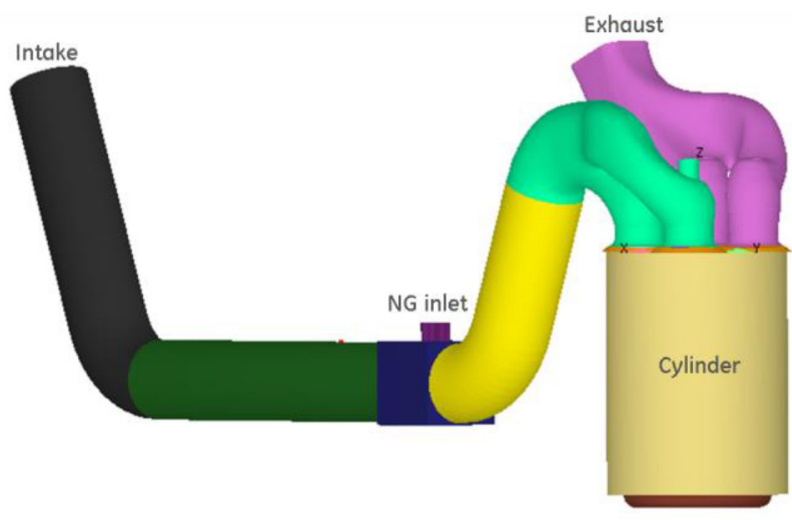

a.

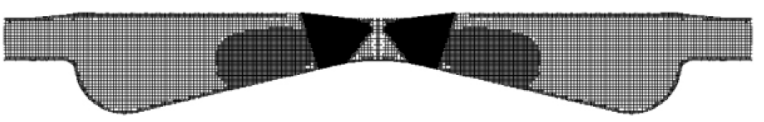

b.

Figure 1. (a) Computational domain showing cylinder with intake and exhaust manifolds and NG inlet in intake manifold, (b) A cut plane showing the computational grid for 2 nozzle holes (out of 8 in the full cylinder simulation domain) during injection.

\section{Combustion Model}

CONVERGE has an integrated chemistry solver, referred to as SAGE, which performs the detailed chemistry calculations on each computational cell. The species composition within a computational cell is assumed to be uniform. The use of AMR with small enough cell sizes $(0.25 \mathrm{~mm})$ in the flame region adequately resolves the turbulent flame front and the species gradients without the need of any sub-grid model. It has been shown previously that the turbulence-chemistry interaction term is typically smaller than the sub-grid term and hence can be neglected, provided the grid is adequately refined to resolve the turbulent flame front [10]. This method has been used before as thickened flame models where increased diffusivity (from RANS model in the current work) is used to thicken the flame and hence resolve it without a sub-grid chemical closure [15]. To correct the flame propagation speed due to this thickening, the turbulent Schmidt number is changed, which affects species diffusion. However, in the present study, the smallest cell size is limited to $0.7 \mathrm{~mm}$ due to heavy computational cost involved for simulating series of multi-cycle full cylinder simulations. The effect of taking coarse mesh $(0.7 \mathrm{~mm})$ in the flame region will be quantified in the future studies.

Performing detailed chemistry calculations on each mesh cell can be computationally expensive. To accelerate the computations, the adaptive-zoning strategy Multi-Zone in $[\underline{16}, \underline{17}, \underline{18}]$ was utilized. In this strategy, cells having similar temperature and composition are grouped into zones, and the chemistry calculations are conducted on zones (based on zone-averaged values of pressure, temperature and species concentrations) rather than on individual cells. The number of zones is fewer than the number of cells, resulting in a significant speedup of the chemistry calculations. This zonal cell grouping is conducted at every time step, and hence adaptive-zoning. The use of adaptive-zoning for engine simulations has been validated in the past with the experimental data taken from Sandia optical engine [19].

Typically, temperature and equivalence ratio are used as variables for grouping cells into zones for single-fuel simulations and multi-fuel premixed simulations without differential molecular diffusion. It has also been shown that for multi-fuel non-premixed simulations (such as the diesel-NG combination of this study), it is important to include some of the species mass fractions, in addition to temperature and equivalence ratio, into the zonal grouping strategy [19]. Equivalence ratio alone may not be able to distinguish cells with different fuel compositions and therefore kinetic behavior. Hence there is a need to include specie mass fractions to segregate cells with different kinetic behavior. For dual fuels, the use of mass fraction of one of the fuel species has been proposed by Raju et al. [18].

\section{Liquid Spray Modeling}

Accurate predictions of diesel spray droplet dynamics and breakup are essential for modeling the subsequent vaporization, mixing with the chamber gases, ignition, and combustion phenomena. The simulations shown in this paper employed the "blob" injection method of Reitz and Diwakar [20] in which "parcels" of liquid with a characteristic size equal to the effective nozzle diameter are injected into the computational domain. The atomization of the liquid blobs/ parcels and subsequent droplets was simulated with models based on the Kelvin-Helmholtz (KH) and Rayleigh-Taylor (RT) instability mechanisms without the use of a breakup length [21]. The No Time Counter (NTC) collision method of Schmidt and Rutland [22] was used for the reported simulations. The NTC model allows for a better statistical representation of a spray since more parcels can be used for a given quantity of injected fuel [24]. When collision occurs, the outcome is predicted to be bouncing, stretching separation, reflexive separation, or coalescence based on the conditions at impact [23] . 


\section{Experimental Details}

Experimental data were acquired on a single-cylinder research engine capable of accepting a full-sized power assembly from a GE Evolution locomotive engine. For these tests, the injector and piston crown were modified from the production design. A general description of the key features can be found in Table 1.

Table 1. Single Cylinder Test Engine Parameters

\begin{tabular}{|l|l|}
\hline Engine Speed & $1050 \mathrm{RPM}$ \\
\hline Rated Power \& BMEP & $279 \mathrm{~kW} \& 20.3 \mathrm{bar}$ \\
\hline Bore $\times$ Stroke & $0.250 \times 0.320 \mathrm{~m}$ \\
\hline Displacement & $15.7 \mathrm{~L} /$ cycle \\
\hline
\end{tabular}

The operating condition selected for the CCV study was using premixed natural gas $(\mathrm{NG})$ and direct injected diesel. $\mathrm{NG}$ is modeled using $\mathrm{CH}_{4}, \mathrm{C}_{2} \mathrm{H}_{6}$ and $\mathrm{C}_{3} \mathrm{H}_{8}$ in gas phase. Diesel is modeled using single species surrogate, $\mathrm{C}_{7} \mathrm{H}_{16}$.

\section{Boundary Conditions}

At the air inlet, total pressure, total temperature and species mass fractions are specified. Static pressure, and temperature are specified at the outlet. Velocity at both air inlet and outlet are set to homogeneous Neumann condition. Mass flow rate and temperature are specified at the NG inlet in the intake port. Pressure was set to homogeneous Neumann condition. Fixed wall temperatures are specified for all solid walls. Diesel injection into the cylinder has been specified via measured injection profile. Measured valve lift profiles are used for the motion of intake and exhaust valves.

\section{Results and Discussion}

Measured cylinder pressure data for the DF case is shown in Figure 2. Usually, CCV in the DF case is higher compared to $100 \%$ diesel cases [1]. The objective of the current work is to understand and quantify the effect of stochastic nature of pre-mixing of air and $\mathrm{NG}$, naturally induced stratification in temperature, turbulence and internal residuals from the prior cycle on the $\mathrm{CCV}$ of pressure in the DF case.

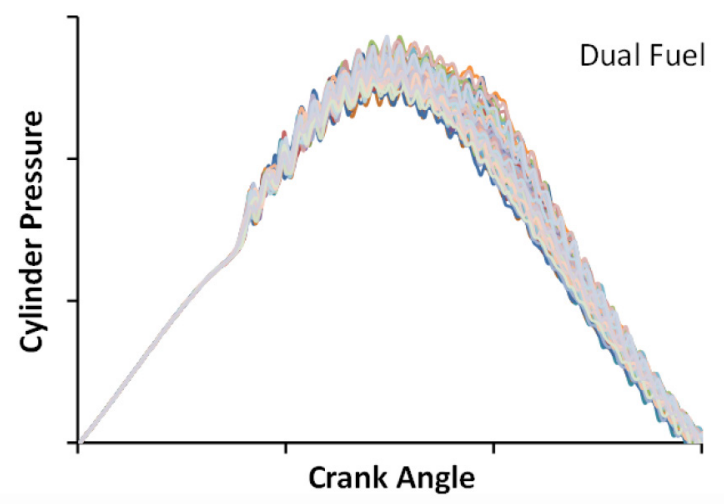

Figure 2. Experimentally measured cycle-to-cycle variation in cylinder pressure for dual-fuel combustion for 100 consecutive cycles

As a first step, a full cycle CFD simulation corresponding to mean operating and boundary conditions was performed to capture the mean pressure trace. The CFD predicted mean pressure trace is shown in comparison with 100-cycle averaged experimental pressure trace in Figure 3. It can be noted that predicted mean pressure matches well with the experimental data. It will be shown in a future publication that the pressure oscillations seen in the experimental data can also be predicted numerically. Local pressure predicted at the same location as the experimental pressure transducer location, at high frequency will show these oscillations.

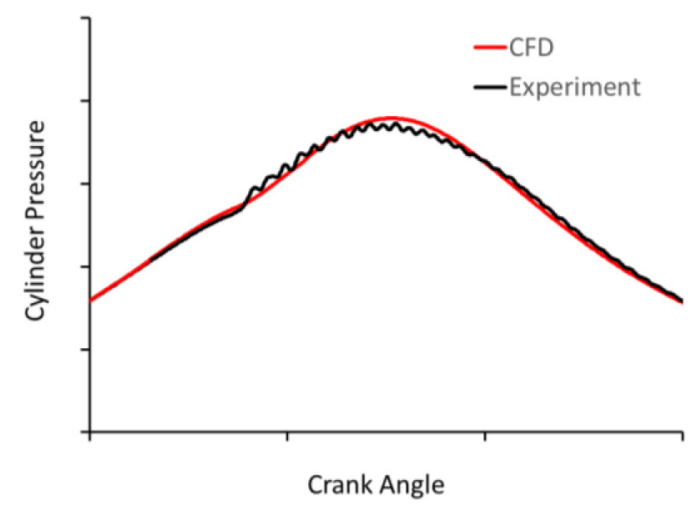

Figure 3. Comparison of CFD predicted mean pressure trace with experiment in dual-fuel combustion operation.

In Figure 4, nine pressure traces obtained from the sequential cycle computation (black lines) are compared to 100 experimental pressure traces for the dual-fuel case. Computed heat release rates (black lines) from the nine consecutive simulation cycles are also presented in the same figure (secondary y-axis). In nine cycles, second cycle produced maximum heat release rate (HRR) and fifth cycle produced minimum heat release rate (HRR). The sequence of peak HRR in nine cycles is shown in Figure 4 next to HRR plot in the decreasing order of cycle number. Contrary to expectation, the magnitude of cycle to cycle variation in the pressure traces was sustained throughout the series of nine cycles calculation. This can be attributed to the stratification in NG, turbulence and temperature in the cylinder. More analysis on the cycle to cycle variation of these parameters is presented in the sequel. The spatial and temporal stratification in a cycle depends on the combustion in the prior cycles. While predicted CCV clustered around the peak firing pressure, $\mathrm{CCV}$ in experiments show variability all the way from the start of combustion to several crank angles in the expansion stroke.

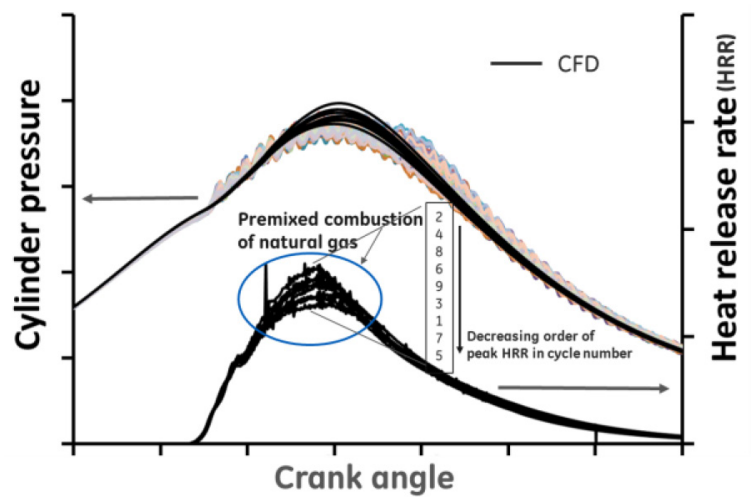

Figure 4. Comparison of CFD predicted CCV (black colored lines) with CCV in experiment (multi-colored lines) in dual-fuel combustion operation.

In Figure 5a, normalized methane $\left(\mathrm{CH}_{4}\right)$ at IVC (blue line) is compared with normalized maximum cylinder pressure $\left(\mathrm{P}_{\max }\right)$ (orange line) and normalized cumulative heat release (grey line) from the nine cycles. $\mathrm{CH}_{4}$ is normalized with respect to maximum mass of $\mathrm{CH}_{4}$ at IVC observed in the nine full cycle calculations. Similarly, $\mathrm{P}_{\max }$ is normalized by the maximum value for $\mathrm{P}_{\max }$ from the nine 
cycles and the heat release is normalized in the same manner. The maximum variation in $\mathrm{CH}_{4}$ at IVC is around $0.5 \%$ of the maximum value. The variation pattern of $\mathrm{CH}_{4}$ at IVC matches the variation of heat release up to six cycles and with $\mathrm{P}_{\max }$ up to five cycles. This indicates that any variation of $\mathrm{CH}_{4}$ mass has introduced variation in the combustion of natural gas and in turn resulted in variation of $\mathrm{P}_{\max }$ and heat release. Further, since all cycles did not follow the same pattern, this also indicates that other factors in addition to $\mathrm{CH}_{4}$ are influencing the $\mathrm{CCV}$ of in-cylinder pressure.

As shown in Figure $5 \mathrm{~b}$ and $\underline{5 \mathrm{c}}$, normalized $\mathrm{O}_{2}$ (blue line), normalized pressure (blue line) and normalized mean temperature, $\mathrm{T}_{\text {mean }}$ (green line) at IVC remain almost constant over all the nine cycles. On the other hand, there is variation of up to $0.35 \%$ in normalized pressure at IVC and this does not correlate with the variation pattern of $\mathrm{P}_{\max }$ and heat release. This is another indication that multiple factors are influencing the $\mathrm{CCV}$ of in-cylinder pressure.

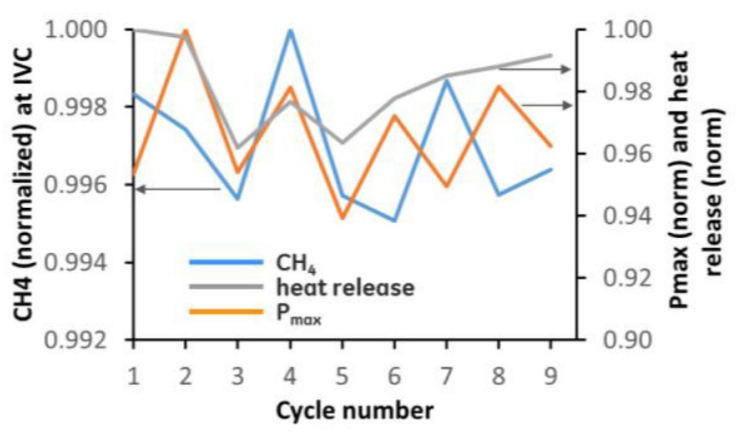

a.

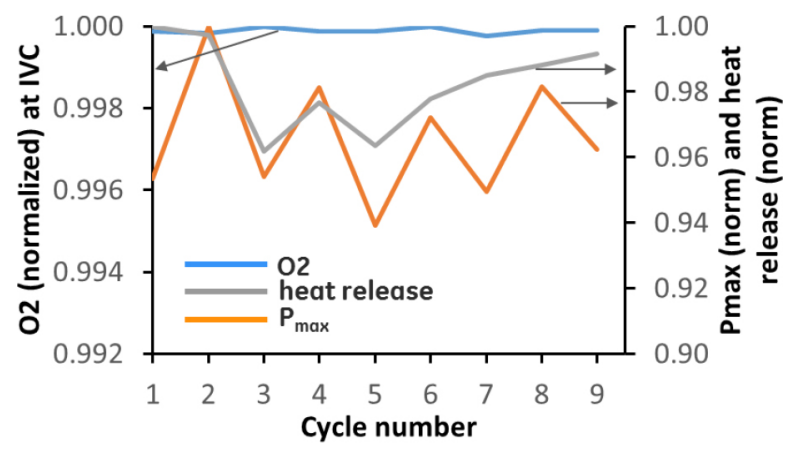

b.

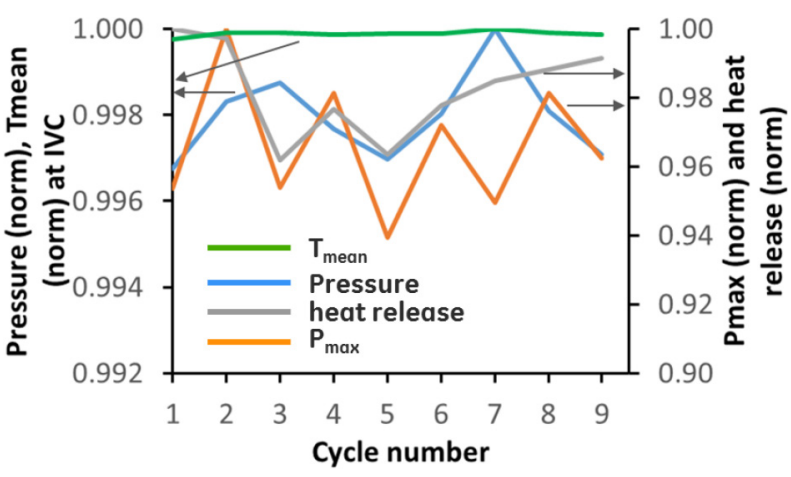

c.

Figure 5. (a) normalized $\mathrm{CH}_{4}$ mass at IVC (b) normalized $\mathrm{O}_{2}$ at IVC (c) normalized pressure and normalized temperature, $\mathrm{T}_{\text {mean }}$ at IVC compared with $\mathrm{P}_{\max }$ and heat release from the nine cycles.
Figure 6 shows $\mathrm{CH}_{4}$ variation at EVO (blue line), peak firing pressure, $\mathrm{P}_{\max }$ (orange line) and heat release (grey line). The calculation of percent $\mathrm{CH}_{4}$ variation at EVO is with respect to minimum mass of $\mathrm{CH}_{4}$ at $\mathrm{EVO}$ in a cycle. It is observed that the minimum mass of $\mathrm{CH}_{4}$ at $\mathrm{EVO}$ is predicted in cycle1.

As observed in Figure 6, the variation pattern in $\mathrm{CH}_{4}$ at EVO matches with heat release variation pattern from cycle to cycle. In a cycle, the low percentage of $\mathrm{CH}_{4}$ at $\mathrm{EVO}$ has given more relative heat release, which means more consumption of $\mathrm{CH}_{4}$ during combustion and more combustion heat release. This confirms that the nine full cycle simulations in series have captured the cycle to cycle variation and is not a numerically induced fluctuation in the observed variables. On the other hand, the $\mathrm{P}_{\max }$ (norm) variation pattern matches with the heat release (norm) pattern up to six cycles and thereafter no correlation is seen between the variables. More insight is provided by quantifying stratification in $\mathrm{CH}_{4}$-air mixing and temperature using probability density function (pdf) plots in the next section.

Stratification in equivalence ratio $(\phi)$ is visualized by plotting the pdf of $\phi$ at each cycle. The difference in stratification between the cycles is quantified by calculating standard deviation $(\sigma)$ for each pdf.

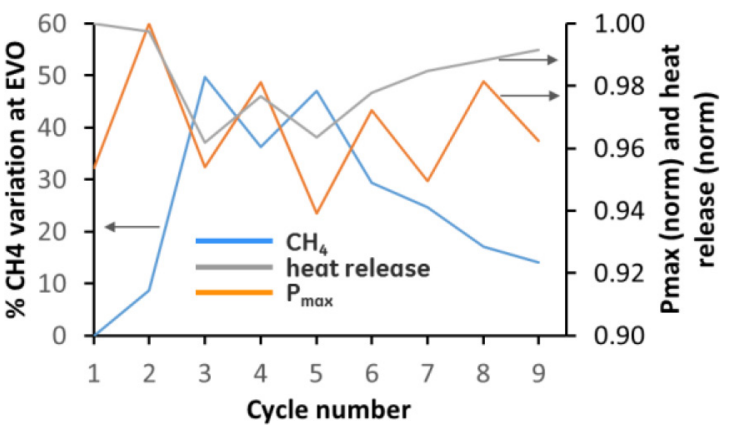

Figure 6. $\mathrm{CH}_{4}$ variation at $\mathrm{EVO}, \mathrm{P}_{\max }$ (norm) and heat release (norm) from nine cycles in series.

Probability density function distributions of equivalence ratio, $\phi$ at IVC for nine cycles are shown in Figure 7a. In Figure 7c, the standard deviation of pdf of $\phi$ (blue line) is compared with $\mathrm{P}_{\max }$ (orange line) and heat release (grey line). As observed, there is stratification in $\phi$, which means mixing of $\mathrm{CH}_{4}$ and air at IVC is not homogenous. The variation of $\sigma_{\phi}$ from cycle to cycle signifies that stratification is changing from cycle to cycle with maximum variation of $15 \%$ with respect to the maximum value of $\sigma_{\phi}$. Also, the variation pattern of $\sigma_{\phi}$ does not have monotonous agreement with the variation of $\mathrm{P}_{\max }$ and heat release. This highlights the fact that not only stratification in mixing but also fluctuation in the mass of $\mathrm{CH}_{4}$ at IVC are affecting the CCV of pressure.

At SOI of diesel, where $\mathrm{CH}_{4}$ stratification in the air influences how the premixed combustion of natural gas occurs after the auto-ignition of diesel, the pdf of $\phi$ and $\sigma_{\phi}$ at SOI of diesel (Figures 7b and $\underline{7 d}$ ) show that there is stratification in $\mathrm{CH}_{4}$ distribution. Almost constant $\sigma_{\phi}$ from second cycle signifies that the cycle to cycle variation is minimal.

In Figures $8 \mathrm{a}$ and $\underline{8 \mathrm{~b}}$, the pdf of temperature at two different crank angle positions within the cycle, IVC and SOI of diesel are shown. The corresponding $\sigma$ of the pdf of temperature plots for these data are shown in Figures 8c and $\underline{8 d}$. Coloring of temperatures in Figure 9 is maintained the same as in Figure 8 . 


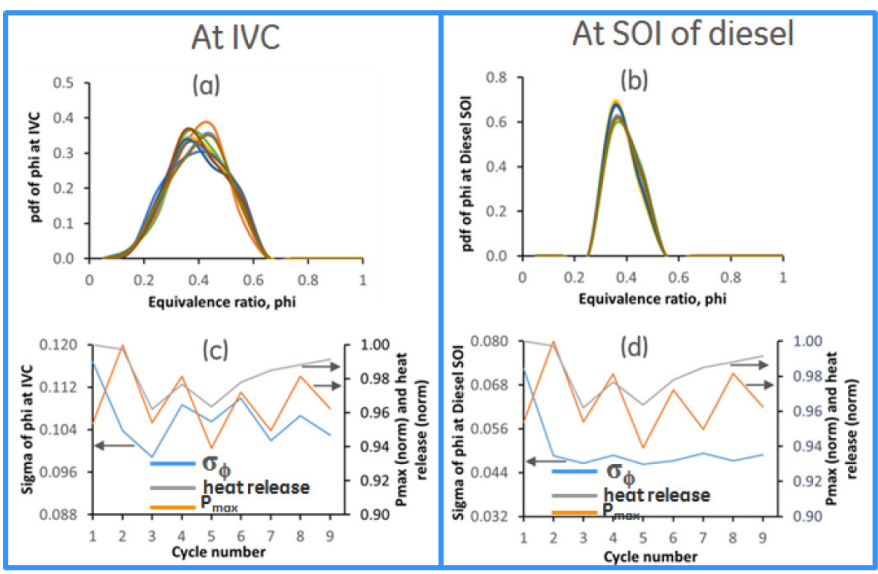

Figure 7. First row: (a) pdf distribution of equivalence ratio $(\phi)$ at IVC for nine cycles. (b) pdf distribution of $\phi$ at SOI of diesel for nine cyclesSecond row: (c) Standard deviation of each pdf at IVC compared with $\mathrm{P}_{\max }$ (norm) and heat release (norm) for nine cycles (d) Standard deviation of each pdf at SOI of diesel compared with $\mathrm{P}_{\max }$ (norm) and heat release (norm) for nine cycles.

There is stratification in temperature both at IVC and SOI of Diesel. The maximum variation in $\sigma$ at IVC is $9 \%$ with respect to maximum $\sigma$ at IVC whereas at SOI of diesel, the value is $20 \%$. Also, correlation does not exist between the $\sigma$ variation and $\mathrm{P}_{\max }$ and heat release both at IVC and SOI of diesel. This signifies the fact that temperature stratification is one of the factors for the predicted CCV.

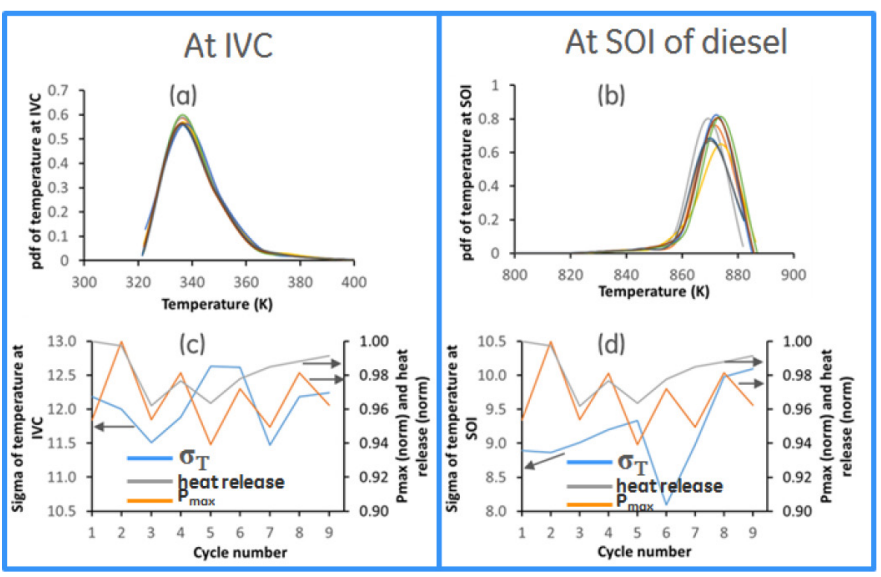

Figure 8. First row: (a) pdf distribution of temperature at IVC for nine cycles. (b) pdf distribution of temperature at SOI of diesel for nine cyclesSecond row: (c) Standard deviation of each pdf at IVC compared with $\mathrm{P}_{\max }$ (norm) and heat release (norm) for nine cycles (d) Standard deviation of each pdf at SOI of diesel compared with $\mathrm{P}_{\max }$ (norm) and heat release (norm) for nine cycles.

Figure 9 shows a comparison of the COV of in-cylinder pressure as a function of crank angle during the main combustion event from the current study, from experimental data and from the earlier work [1]. In [1], several parallel closed cycle simulations were performed with perturbations on boundary and initial conditions. It can be observed from Figure 9, that the COV predicted from the present sequential full cycle simulations is closer to the experiment than that from the previous work reported in [1]. The predicted peak value of COV is 2.1 which occurs at 11 crank angle degrees ATDC. However, in the experiments, the peak value of COV is 3.3 and it occurs at 24 crank angle degrees ATDC. In the parallel closed cycle simulations, the peak is observed at a few crank angles downstream compared to the current prediction. This trend gives an indication that combining the stochastic stratification effects and the deterministic perturbations in boundary and initial conditions in full cycle simulations may bring the COV closer to the one observed in the experiment. Moreover, Large Eddy Simulation (LES) may capture COV in a better way compared to RANS based simulations.

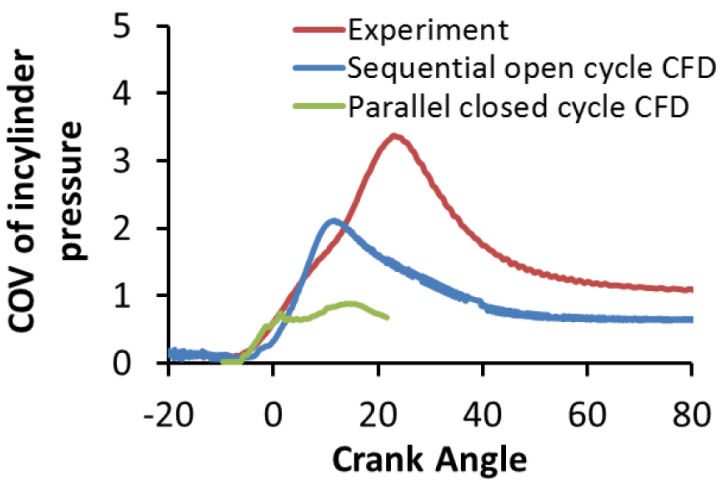

Figure 9. COV of pressure predicted from sequential open cycle CFD study (blue line) compared with experiment (red line) and parallel closed cycle CFD from [1] (green line)

\section{Conclusions}

Dual-fuel operation under certain operating conditions can lead to high cycle-to-cycle variability (CCV) in the combustion event, resulting in variations in cylinder pressure and work output. High CCV can limit the NG substitution rate and operating range of the dual-fuel engine by increasing emissions and reducing engine stability, reliability and fuel efficiency via incomplete natural-gas combustion. It is important to understand the factors causing high $\mathrm{CCV}$. In the current study, nine full cycle simulations have been performed in series to capture the effect of in-cylinder stratifications in (a) natural gas, (b) temperature and (c) turbulence without perturbing or varying the initial and boundary conditions. Following are the key observations from the nine full cycle simulations in series.

1. Among the nine cycles, $\mathrm{CH}_{4}$ mass at IVC is found to vary from cycle to cycle. However, the maximum variation is found to be in the order of $0.5 \%$. This variation pattern in $\mathrm{CH}_{4}$ mass matched with heat release pattern up to six cycles and with $\mathrm{P}_{\max }$ up to five cycles. On the other hand, the pressure variation at IVC does not correlate with heat release and $\mathrm{P}_{\max }$ variation. Variation in $\mathrm{T}_{\text {mean }}$ and $\mathrm{O}_{2}$ are found to be negligible.

2. At EVO, the unburned $\mathrm{CH}_{4}$ mass correlates with $\mathrm{CCV}$ in heat release rate over all nine cycles. This suggests that the predicted $\mathrm{CCV}$ is not a numerical induced fluctuation but that it is driven by the variation in combustion pattern from cycle to cycle.

3. At IVC, the pdf of $\phi$ and temperature showed that there is stratification in both. Their standard deviations varied up to $15 \%$ of their maximum values. At SOI of diesel, $\sigma_{\phi}$ remained flat over all the cycles and $\sigma$ of temperature varied up to $20 \%$ with respect to its peak value. Both the stratifications do not correlate with $\mathrm{CCV}$ in heat release rate and $\mathrm{P}_{\max }$.

4. No single variable is found to correlate fully with the CCV of $\mathrm{P}_{\max }$ and heat release rate. This highlights the fact that the $\mathrm{CCV}$ is the combined effect of $\mathrm{CH}_{4}$ mass variation at IVC, stratification in natural gas, temperature and turbulence both at IVC and SOI of diesel. 
5. The predicted peak value of COV of in-cylinder pressure by continuous series of nine full cycle simulations is 2.1 compared to 3.3 in the experiment. The open cycle predicted COV variation is found to be better than the corresponding prediction from parallel closed cycle simulations [1] .

\section{References}

1. Jupudi, R., Finney, C., Primus, R., Wijeyakulasuriya, S. et al., "Application of High Performance Computing for Simulating Cycle-to-Cycle Variation in Dual-Fuel Combustion Engines," SAE Technical Paper 2016-01-0798, 2016, doi:10.4271/2016-01-0798.

2. Hountalas, D. and Papagiannakis, R., "A Simulation Model for the Combustion Process of Natural Gas Engines with Pilot Diesel Fuel as an Ignition Source," SAE Technical Paper 200101-1245, 2001, doi:10.4271/2001-01-1245.

3. Saha, K., Som, S., Li, Y., Quan, S. and Battistoni, M., "Modeling of Internal and Near-nozzle Flow for a GDI Fuel Injector", ICEF2015-1112, 2015.

4. Klos, D. and Kokjohn, S.L., "Investigation of the Effect of Injection and Control Strategies on Combustion Instability in Reactivity Controlled Compression Ignition (RCCI) Engines", Journal of Engineering for Gas Turbines and Power, 138(1), 2015.

5. Kokjohn, S.L., Musculus, M.P.B. and Reitz, R.D., "Evaluating Temperature and Fuel Stratification for Heat-Release Rate Control in a Reactivity-Controlled Compression-Ignition Engine using Optical Diagnostics and Chemical Kinetics Modeling”, Combustion and Flame, Vol. 162, No. 6, 2015.

6. Kokjohn, S., Reitz, R., Splitter, D., and Musculus, M., "Investigation of Fuel Reactivity Stratification for Controlling PCI Heat-Release Rates Using High-Speed Chemiluminescence Imaging and Fuel Tracer Fluorescence," SAE Int. J. Engines 5(2):248-269, 2012, doi:10.4271/2012-01-0375.

7. Richards K.J., Senecal P.K. and Pomraning E. 2015, "CONVERGE (Version 2.2) Manual," Convergent Science, Inc., Madison, Wisconsin, USA.

8. Issa, R.I., "Solution of the Implicitly Discretized Fluid Flow Equations by Operator-Splitting," Journal of Computational Physics, 62(1): 40-65, 1986.

9. Yakhot, V., Orszag, S.A., Thangam, S., Gatski, T.B. and Speziale, C.G., "Development of turbulence models for shear flows by a double expansion technique," Physics of Fluids A, 4(7): 1510-1520, 1992.

10. Pomraning, E., Richards, K., and Senecal, P., "Modeling Turbulent Combustion Using a RANS Model, Detailed Chemistry, and Adaptive Mesh Refinement," SAE Technical Paper 2014-01-1116, 2014, doi: 10.4271/2014-01-1116.

11. Som, S., Longman, D., Aithal, S., Bair, R. et al., "A Numerical Investigation on Scalability and Grid Convergence of Internal Combustion Engine Simulations," SAE Technical Paper 201301-1095, 2013, doi:10.4271/2013-01-1095.

12. Scarcelli, R., Sevik J. Jr., Wallner Thomas, Richards, K. and Senecal, P., "Capturing Cyclic Variability in EGR Dilute SI Combustion using Multi-Cycle RANS," ICEF2015-1045, 2015.
13. Wang, Z., Scarcelli, R., Som, S., McConnell, S. et al., "MultiDimensional Modeling and Validation of Combustion in a HighEfficiency Dual-Fuel Light-Duty Engine," SAE Technical Paper 2013-01-1091, 2013, doi:10.4271/2013-01-1091.

14. Wijeyakulasuriya, S. D., Givler, S., Raju. Mandhapati, Ravichandra S. J., Ashwin R., Primus Roy J, Klingbeil Adam E., "Multidimensional Modeling and Validation of Dual-Fuel Combustion in a Large Bore Medium Speed Diesel Engine," ICEF2015-1077, 2015.

15. Colin, O., Ducros, F., Veynante, D., Poinsot, T., “A thickened flame model for large eddy simulations of turbulent premixed combustion," Physics of Fluids, Vol. 12, No. 7, pp. 18431863, 2000 .

16. Babajimopoulos, A.D., Assanis, N.D., Flowers, L.S., Aceves, M.R. and Hessel, P., "A Fully Coupled Computational Fluid Dynamics and Multi-Zone Model with Detailed Chemical Kinetics for the Simulation of Premixed Charge Compression Ignition Engines, ' International Journal of Engine Research, 6(5): 497-512, 2005.

17. Lucchini, T., D’Errico, G., Ettorre, D., and Ferrari, G., "Numerical Investigation of Non-Reacting and Reacting Diesel Sprays in Constant-Volume Vessels," SAE Int. J. Fuels Lubr. 2(1):966-975, 2009, doi:10.4271/2009-01-1971.

18. Raju, M., Wang, M., Dai, M., Piggott, W. et al., "Acceleration of Detailed Chemical Kinetics Using Multi-zone Modeling for CFD in Internal Combustion Engine Simulations," SAE Technical Paper 2012-01-0135, 2012, doi:10.4271/2012-01-0135.

19. Senecal, P.K., Pomraning, E., Anders, J.W., Weber, M.R., Gehrke, C.R., Polonowski, C.J. and Mueller, C. J., "Predictions of Transient Flame Lift-off Length with Comparison to SingleCylinder Optical Engine Experiments", Journal of Engineering for Gas Turbines and Power, 136(11): 111505-111505-19, 2014.

20. Reitz, R. and Diwakar, R., "Structure of High-Pressure Fuel Sprays," SAE Technical Paper $\underline{870598,1987,}$ doi:10.4271/870598.

21. Senecal, P., Richards, K., Pomraning, E., Yang, T. et al., "A New Parallel Cut-Cell Cartesian CFD Code for Rapid Grid Generation Applied to In-Cylinder Diesel Engine Simulations," SAE Technical Paper 2007-01-0159, 2007, doi:10.4271/2007-01-0159.

22. Schmidt, D.P. and Rutland, C.J., "A New Droplet Collision Algorithm," Journal of Computational Physics, 164(1): 62-80, 2000.

23. Senecal, P.K., Pomraning, E., Richards, K.J. and Som, S., "GridConvergent Spray Models for Internal Combustion Engine CFD Simulations," Journal of Energy Resources Technology, 136(1): 012204-012204-11, 2014.

24. https://www.hpc.kaust.edu.sa

\section{Contact Information}

Dr. Ravichandra S Jupudi

GE Global Research, 122, EPIP, Phase 2, Hoodi Village, Whitefield Road, Bangalore 560066

Ravichandra.js@ge.com 


\section{Acknowledgments}

This research used resources of the KAUST Supercomputing

Laboratory located at King Abdullah University of Science and

Technology, Saudi Arabia.

Components of this work were supported by the U.S. Department of Energy, Vehicle Technologies Office.

\section{Definitions/Abbreviations}

AMR - Adaptive mesh refinement

CCV - Cycle to cycle variation

CFD - Computational fluid dynamics

CNG - Compressed natural gas

COV - Coefficient of variation

DF - Dual fuel

EVO - Exhaust Valve Open

GDI - Gasoline direct injection

IMEP - Indicated mean effective pressure
IVC - Intake valve close

KH - Kelvin-Helmholtz

NG - Natural Gas

LNG - Liquefied natural gas

MMBTU - Million BTU

MPI - Message passing interface

NTC - No-Time-Counter

RANS - Reynolds Averaged Navier Stokes

RNG - renormalization group

RT - Rayleigh-Taylor breakup

SOI - Start of Injection

\section{Nomenclatures}

pdf - Probability density function

$\phi$ - Equivalence ratio

$\boldsymbol{\sigma}$ - Standard deviation

$\boldsymbol{\sigma}_{\phi}$ - Standard deviation of pdf of equivalence ratio

$\boldsymbol{\sigma}_{\mathrm{T}}$ - Standard deviation of pdf of temperature

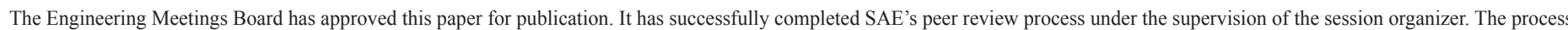
requires a minimum of three (3) reviews by industry experts.

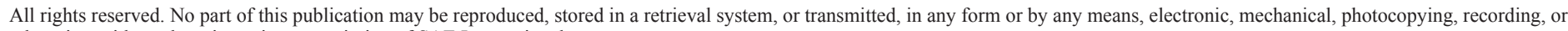
otherwise, without the prior written permission of SAE International.

Positions and opinions advanced in this paper are those of the author(s) and not necessarily those of SAE International. The author is solely responsible for the content of the paper.

ISSN 0148-7191

http://papers.sae.org/2017-01-0772 